\title{
Facile Method for Constructing Metallic Nanoarrays on a Solid Surface
}

\author{
Hidenobu NAKaO \\ Nanoarchitecture Group, Organic Nanomaterials Center, National Institute for Materials Science, \\ 1-1 Namiki, Tsukuba, Ibaraki 305-0044, Japan
}

\begin{abstract}
This review highlights work using the author's method to construct metallic nanoarrays. This method is a DNA-templated assembly, to assemble metal nanoparticles (MNPs) in which DNA molecules are initially stretched and aligned on surfaces. Simple interface (air-liquid) moving and surface coating with polymers containing $\pi$-conjugation units, lead to the controlled formation of one-dimensional (1D) nanoarrays of DNA without special equipment. The assembly of MNPs onto DNA can be driven by electrostatic binding of newly prepared gold nanoparticles with positive charges, leading the formation of 1D metallic nanoarrays. Dark-field microscopy observations indicate that MNPs on DNA are uniaxially aligned. Finally, the fabrication and patterning of metallic nanoarrays achieved with DNA nanofibers and transfer printing techniques are discussed.
\end{abstract}

(Received October 5, 2009; Accepted October 20, 2009; Published December 10, 2009)

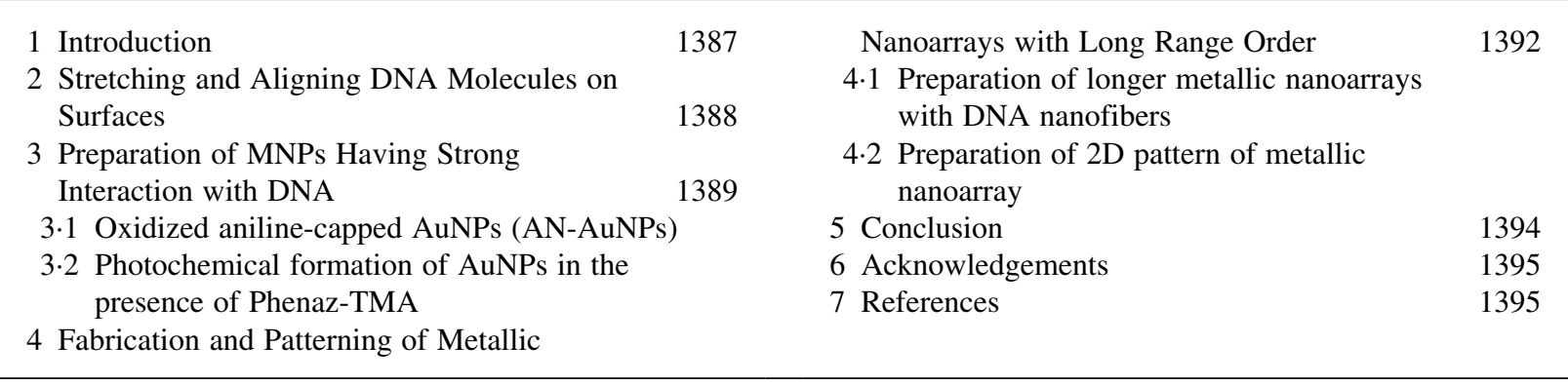

\section{Introduction}

Metal nanoparticles (MNPs) such as Au or Ag can confine light (electromagnetic fields) to their dimensions on the order of or smaller than the wavelength. Such properties, called localized plasmon resonances (LPRs), are based on interactions between electromagnetic radiation and conduction electrons at metallic interfaces or in metallic nanostructures, leading to an enhanced optical near field of sub-wavelength dimension below the diffraction limit. ${ }^{1}$ LPRs of MNPs are tunable throughout the visible and near-infrared region of the spectrum as a function of the particle size, shape, aggregation state, and local environment. ${ }^{1}$ When MNPs are organized in closely spaced arrays, their LPR peak is shifted towards low energy, and an increase in the bandwidth is observed because the dielectric constant of the surrounding MNPs is increased. ${ }^{1-3}$ Molecules absorbed by the surface of the mentioned array undergo a surface-enhanced Raman scattering (SERS) effect, enhancing its Raman signal. ${ }^{4-6}$ This process has attracted much attention due to its potential applications in miniaturized optical devices, sensors and single molecule detection via SERS. ${ }^{7-11}$ Therefore, creating periodic

E-mail: nakao.hidenobu@nims.go.jp assembled MNP structures through this unique innovative approach has attracted much attention lately.

In general, MNPs can be organized using bottom-up approaches based on self-assembly. Simple self-assembly strategies introduce functional groups (ligands) onto MNPs, as specific physical and chemical affinities such as covalent or non covalent (van der Waals, hydrophobic, or electrostatic) interactions that can be exploited to achieve the precise self-assembly of MNPs. The chemisorption of alkanethiols and other organosulfur molecules on $\mathrm{Au}$ or $\mathrm{Ag}$ nanostructures has been widely applied because of their ability to form a well-organized self-assembled monolayer. ${ }^{12}$ Typically, the adsorption and desorption of ligands depend on the type of crystallographic facet involved. With alkanethiol molecules, preferential binding of thiols to the $\{111\}$ surface is obtained. ${ }^{13,14}$ Using this approach, the facet-selective assembly of MNPs can be achieved, leading to one-dimensional (1D) chains of MNPs. In particular, since metal nanorods with a high aspect ratio $(\sim 25)$ have $\{111\}$ at each end and $\{100\}$ at their sides, the end-to-end assembly of metal nanorods is oriented in the same direction. ${ }^{7-9}$ Such a 1D array of metal nanorods strongly absorbs light of specific wavelengths with polarizations parallel to the rod axis. ${ }^{1-3}$

Precise assembly and patterning of MNPs on surfaces and 
interfaces are important in the next phase for the applications described above. Current lithography methods and microcontact printing can either directly produce arrays of $\mathrm{MNP}^{2,3,15}$ or predefine a surface (geometrically, chemically, or electrostatically) to assist the assembly of MNPs on a surface. ${ }^{16-19}$ In different MNP assembly approaches, interfaces such as gas-solid and liquid-liquid play important roles. The Langmuir-Blodgett technique is a typical interfacial assembly process. ${ }^{20-22}$ Surface-protected MNPs are generally made to float over a water surface using hydrophobic interactions. At the water-air interface, MNPs form a monolayer, which is slowly compressed and transferred during the compression process, using horizontal or vertical lift-off, to substrates such as silicon. Using this technique, 1D arrays or films of various well-ordered MNPs can be achieved. ${ }^{23}$ Ordered arrangements of MNPs on templates such as block copolymer scaffolds ${ }^{24}$ and along crystal step edges ${ }^{25,26}$ have also been demonstrated. Many natural materials that have specific binding properties in molecular recognition and self-assembly can be used as templates to organize and interconnect assemblies of MNPs on surfaces. ${ }^{25-30}$ DNA in particular has been widely investigated as a template because of its structurally controlled nanowire of $2 \mathrm{~nm}$, well-defined polymeric sequence and many functionalities, which make possible a 1D array of MNPs. ${ }^{31-36}$ Although these methods involve assemblies of MNPs, they often require complex processes and specific equipment.

In this review, we highlight our efforts to produce highly aligned 1D arrays of MNPs (metallic nanoarrays) on surfaces. We aim to present a simple and reproducible method for creating metallic nanoarrays without any special equipment. In our assembly of MNPs, highly aligned DNA molecules or nanofibers on a surface are used as a 1D template and can be driven by simple interface (air-liquid) movement. Positively charged MNPs can be explored to produce MNPs that have strong binding properties with DNA strands, leading to highly aligned 1D metallic nanoarrays on surfaces. Furthermore, the preparation and patterning of metallic nanoarrays with long-range order are presented using DNA nanofibers and transfer-printing (TP) techniques. Finally, optical applications that take advantage of light fields localized on prepared metallic nanoarrays are discussed.

\section{Stretching and Aligning DNA Molecules on Surfaces}

DNA has a well-defined structure for the bottom-up construction of artificial nanostructures and networks in one, two, and three dimensions. ${ }^{37-43}$ In particular, the specificity of Watson-Crick base pairing enable the programming of its intramolecular and intermolecular associations, then making use of its unique molecular recognition and self-assembly capabilities to construct DNA-based architectures. Having a diameter of $2 \mathrm{~nm}$ and a virtually infinite length, providing a large variety of binding sites for different metal ions, and having remarkable mechanical properties, DNA constitutes an ideal template for the organization of metallic and semiconductor nanoparticles into 1D assemblies. Metallic wires grown on DNA have been observed to possess peculiar transport properties both at room temperature and at low temperature; thus, they are good candidates for opto-electronic applications.

Since DNA molecules in solution have the conformation of a random coil, they must be stretched and aligned on surfaces to create templates of 1D arrays. Generally, DNA molecules have been stretched and aligned at macroscopic or single molecule

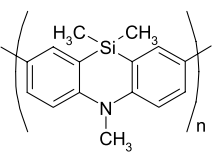

Polyphenazasiline (PPhenaz)

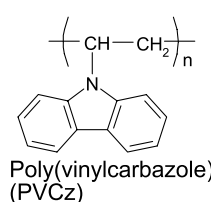

(PVCz)

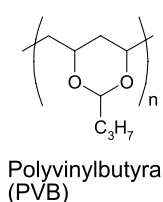

(PVB)

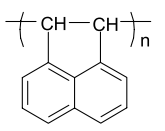

Polyacenaphtylene

(PA)

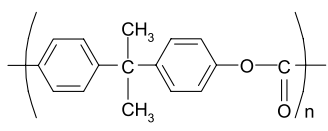

Polycarbonate (PC)

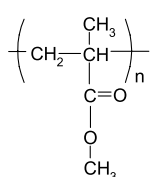

$\stackrel{\mathrm{I}}{\mathrm{CH}_{3}}$ Poly(methyl methacrylate) (PMMA)
Fig. 1 Chemical structures of polymers in this study.

The polymer solution $(50 \mu \mathrm{L})$ was deposited on

the coverslip and spin-coated for $20 \mathrm{~s}$ at

$5000 \mathrm{rpm}$.

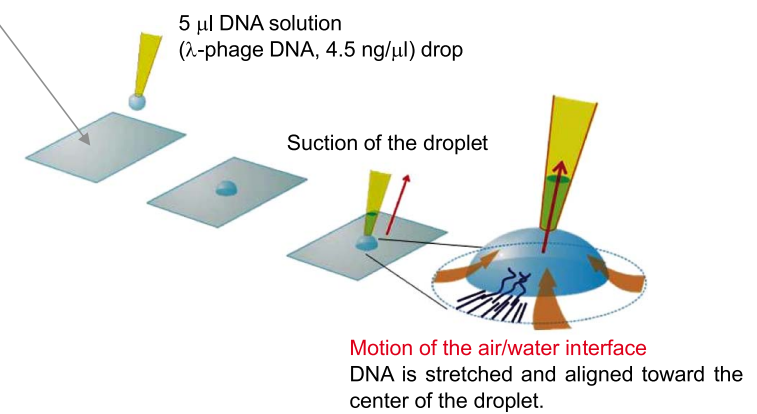

Fig. 2 Procedure of DNA stretching and alignment on surfaces.

level by hydrodynamic flow, ${ }^{4-47}$ meniscus forces, ${ }^{48-52}$ electric-field, ${ }^{53,54}$ atomic force microscope (AFM), ${ }^{55,56}$ optical trapping, ${ }^{57}$ or magnetic tweezers. ${ }^{58}$ Surface modifications, such as silanization ${ }^{48-51}$ and poly-L-lysine ${ }^{47}$ modification of substrate surfaces have also been utilized to facilitate the fixation and stretching of DNA molecules using the above methods. Though controlling the interaction between DNA molecules and surfaces is very important for stretching and aligning DNA molecules, the interaction mechanism at the chemical structure level has not been sufficiently investigated.

We have reported a useful technique for reproducibly stretching and aligning DNA on surfaces..$^{59}$ The point of this technique is to control the interaction between surfaces and DNA using a polymer coating. Chemical structures of the coating polymers selected in this study are presented in Fig. 1. A solution of $5 \mu \mathrm{L}$ of DNA $(4.5 \mathrm{ng} / \mu \mathrm{L})$ in TE buffer $(10 \mathrm{mM}$ Tris- $\mathrm{HCl}$ and $1 \mathrm{mM}$ EDTA, $\mathrm{pH} 8$ ) was deposited on such polymer-coated glass surfaces, and then its droplet was sucked up using pipet. When a droplet of DNA solution on a surface is sucked up, the surface tension at the moving air-water interface is sufficient to stretch and align the molecules along the central direction of the droplet (Fig. 2).

The stretching of DNA on surfaces coated with various polymers was examined. We observed fluorescence microscopic images of DNA deposited on various polymer-coated coverslips. On uncoated surfaces, the observed image revealed that only a few molecules were partially stretched and that many molecules were aggregated. Because the glass surface has strong polar groups, it seems that DNA interacts with the surface strongly 

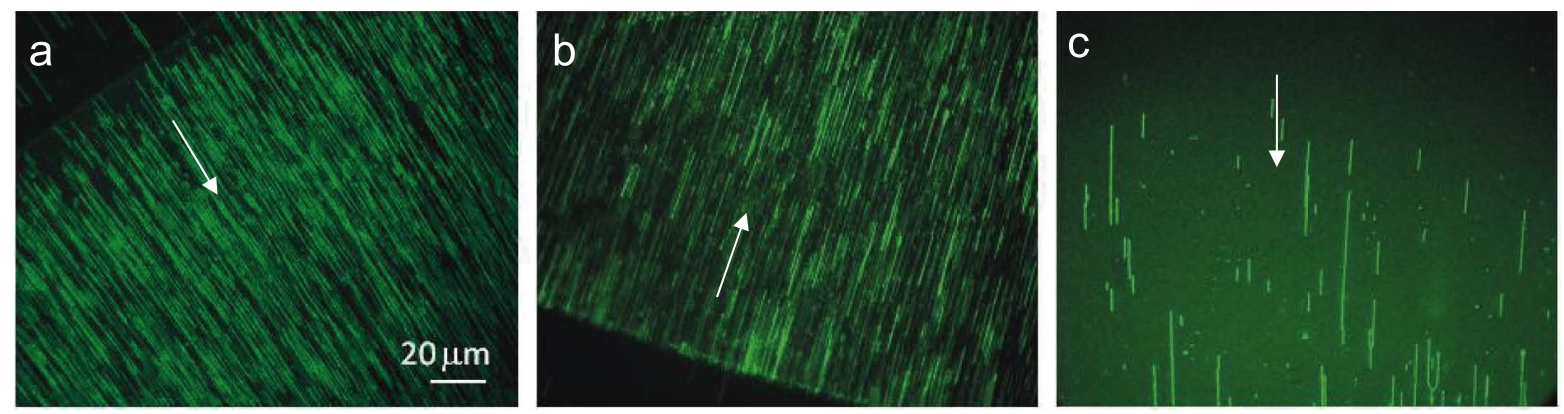

Fig. 3 Fluorescence microscopic images of DNA stretched on various polymer-coated surfaces. PA-coated glass (a). PC-coated glass (b). PMMA-coated glass (c). The arrow shows the central direction of the droplet.

and nonspecifically. However, DNA molecules were not sufficiently fixed on PVB-coated surfaces. PVB-coated glasses have hydrophobic surfaces, so that the interactions of DNA having negative charges or hydrophilic groups with these surfaces are very weak. In contrast, the observed DNA molecules on PVCz- and PPhenaz-coated coverslips were sufficiently fixed and stretched. In addition, these images demonstrate that DNA molecules are stretched and aligned in a central direction of the DNA droplet. Thus, it seems that PVCzand PPhenaz-coated coverslips are useful for depositing and imaging well-stretched DNA. The AFM image of well-stretched DNA on both polymer-coated surfaces could be also observed. Observed images of both surfaces demonstrated that DNA molecules were highly stretched and densely aligned in the same direction. Furthermore, the root mean square (RMS) roughness of the uncoated coverslip decreased from 5.013 to $0.319 \mathrm{~nm}$ after coating with PPhenaz and to $0.512 \mathrm{~nm}$ after coating with PVCz. It should be noted that these polymer coatings enabled obtaining adequate AFM images of DNA on conventional coverslips.

Although PVCz and PPhenaz have a similar hydrophobicity to PVB, DNA molecules sufficiently fixed and stretched on both surfaces. The above results suggest that these polymers have a specific interaction with DNA. To understand the interactions between these polymers and DNA, we examined their absorption spectral properties. When we added DNA molecules to a solution (mixture of TE-buffer $(\mathrm{pH} 8.0)$ :THF $=1: 1)$ containing PPhenaz, the absorption band at $390 \mathrm{~nm}$, which is attributed to the $\pi-\pi^{*}$ transition of PPhenaz, decreased gradually. Such a decrease in the absorbance shows a strong interaction between the electronic state of the polymer and that of the DNA base. Consequently, it seems that these spectral changes are characteristic of an interaction, such as $\pi$-stacking, between aromatic compounds and DNA. ${ }^{60-62}$ We observed a similar phenomenon for PVCz. From a molecular viewpoint, their aromatic amine units are planar, or almost planar, favoring the insertion of polymer units into the hydrophobic interior of the DNA base stack. We therefore assume that DNA attaches to surfaces through $\pi$-stacking between aromatic amines in polymers and base pairs in DNA. In addition, the $\lambda$-phage DNA used in this study was linear DNA with 12-base-long sticky ends, which exposed the hydrophobic $\pi$-core (bases) on both ends of the helix. Since such positions have a strong affinity with surfaces, DNA molecules are preferentially anchored at either end. ${ }^{50}$ Consequently, effective DNA-stretching and fixations are achieved on surfaces. By using some polymers containing $\pi$-units other than aromatic amines, we found that such polymers effectively stretch and fix DNA molecules on surfaces (Fig. 3). Thus, the above results strongly indicate that $\pi-\pi$ interaction is the important factor for DNA-stretching and fixations in our system.

\section{Preparation of MNPs Having Strong Interaction with DNA}

The interaction of MNPs with DNA is a well-known phenomenon, ${ }^{63-66}$ and single or double-strand DNA is decorated by MNPs, which can easily be imaged by transmission electron microscopy (TEM) or AFM. ${ }^{67-69}$ To attach AuNPs securely to DNA molecules, various surface-functionalized AuNPs have been prepared. In numerous studies, such AuNPs were prepared by surface modifications with cationic thiols ${ }^{35,36,69}$ or intercalators..$^{70,71}$ The 1D chains of AuNPs coated with cationic trimethyl(mercaptoundecyl)ammonium monolayers were electrostatically assembled along DNA molecules in solution by the relative molar quantities of AuNPs and DNA base pairs. ${ }^{69}$ Since psoralen acts as a specific intercalator for A-T base pairs, the functionalized AuNPs were assembled onto the pA-pT ds-DNA. ${ }^{70}$ Furthermore, UV irradiations induced reactions between the psoralen units and the thymine of DNA, then covalently fixed AuNPs to the DNA.

\section{3·1 Oxidized aniline-capped AuNPs (AN-AuNPs)}

Recently, we reported a one-step preparation of surface-functionalized AuNPs without ligand exchange. ${ }^{72}$ Novel surface-functionalized AuNPs, "AN-AuNPs" were prepared based on the conventional reduction of $\mathrm{HAuCl}_{4}$ using aniline as a reducer, so that the AN-AuNPs had a positive charge and aromatic ring on the surface (due to the formation of oxidized aniline during preparation), which resulted in their electrostatic binding to the negatively charged phosphate backbone of DNA. Characterization of prepared particles by electrophoresis analysis, zeta-potential measurements and UV measurements revealed the presence of positive charges on their surfaces. We then experimented with assemblies of AN-AuNPs organized on DNA molecules. Two different procedures were used here (Fig. 4). In Method I, DNA was stretched and fixed on the surface according to the above method, which resulted in highly aligned DNA patterns formed on surfaces. Next, DNA molecules were treated with AN-AuNPs solution for $5 \mathrm{~min}$, then rinsed in water. Before treatments with AN-AuNPs, the height (diameter) of DNA molecules imaged by atomic force microscopy (AFM) on the surface was $\sim 1.0 \mathrm{~nm}$, which agreed well with that of a single double-stranded DNA in a previous study. After a treatment with AN-AuNPs, AFM observation 

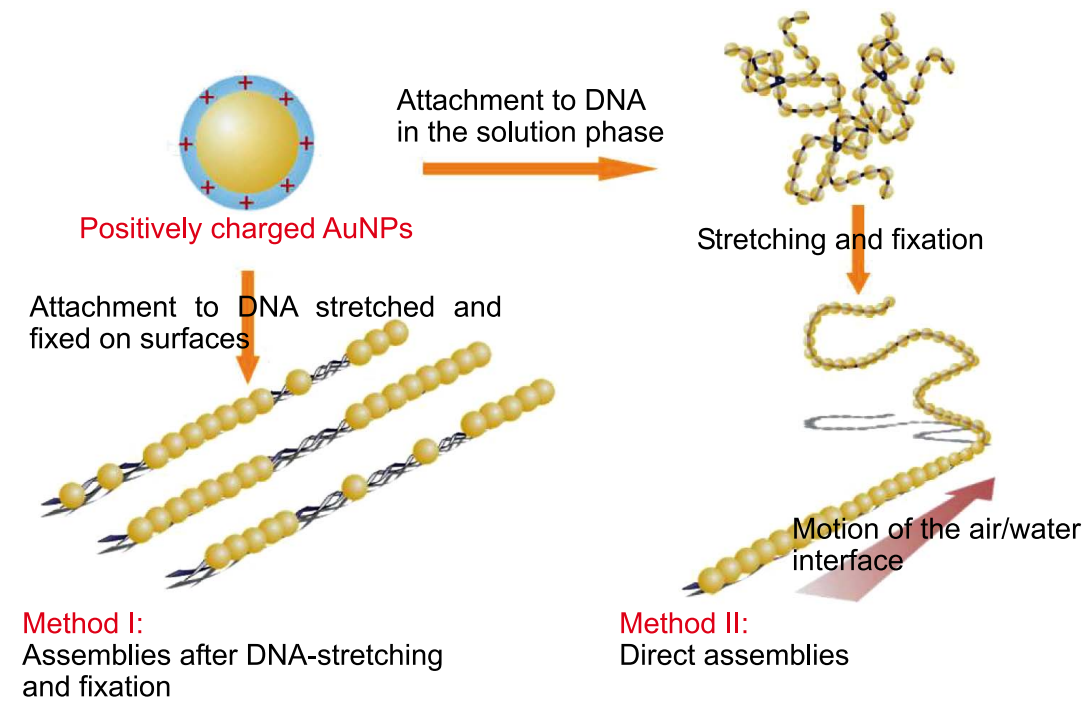

Fig. 4 Procedure of assemblies of ANAuNPs onto DNA.

revealed that many DNA molecules on the surfaces had contiguous particles with raised height, indicating that the observed heights of DNA molecules were $2.14 \pm 0.35 \mathrm{~nm}$. The majority of particles that can be distinguished from the background are $1.56 \pm 0.21 \mathrm{~nm}$. Consequently, it is reasonable that the increased DNA molecule heights after treatment were caused by particle deposition. In method II, we prepared a mixture of an AN-AuNP solution and a $\lambda$-DNA solution, and incubated it for $30 \mathrm{~min}$. Next, samples were stretched and fixed on surfaces according to the above method. It was very interesting to note that AN-AuNPs with larger interparticle spacing were assembled along the DNA molecules in a necklace-like formation. Since the DNA molecules (to which AN-AuNPs were already attached) were stretched significantly by surface tension, the interparticle spacing was greater. Figure 5 depicts AFM images of AN-AuNPs-attached DNA molecules by two methods. By depositing different MNPs in interparticle spacing, it should be possible to tune the electrical or optical properties of linear arrays. Most recently, a one-step process achieved raspberry- ${ }^{73}$ and mushroom-like aggregates ${ }^{74}$ of AN-AuNPs, that have three-dimensional structures on a nano-order level. DNA templates also should be helpful in aligning such aggregates, which produce metallic nanoarrays having significant electrical and optical properties.

\subsection{Photochemical formation of AuNPs in the presence of Phenaz-TMA}

As described above, many DNA molecules were sufficiently stretched and fixed on PPhenaz-coated surfaces by the $\pi-\pi$ interaction between $\pi$-conjugated units in polymer and base pairs in DNA. To enhance the solubility of PPhenaz in a polar solvent, such as water and interactions with DNA having negative charges, PPhenaz having alkylammonium salts on the $\mathrm{N}$ atom (PPhenaz-TMA) were synthesized (Fig. 6), directly forming $\pi$-conjugated polymer functionalized DNA (PPhenaz-TMA/DNA) nanowires. ${ }^{75}$ By utilizing the redox reaction between PPhneaz-TMA and $\mathrm{AuCl}_{4}^{-}$, PPhneazTMA/DNA nanowires could be easily converted to Au deposited nanowires. Consequently, AFM and scanning near-field optical microscope (SNOM) observations revealed that highly aligned metallic nanoarrays were fabricated on DNA.
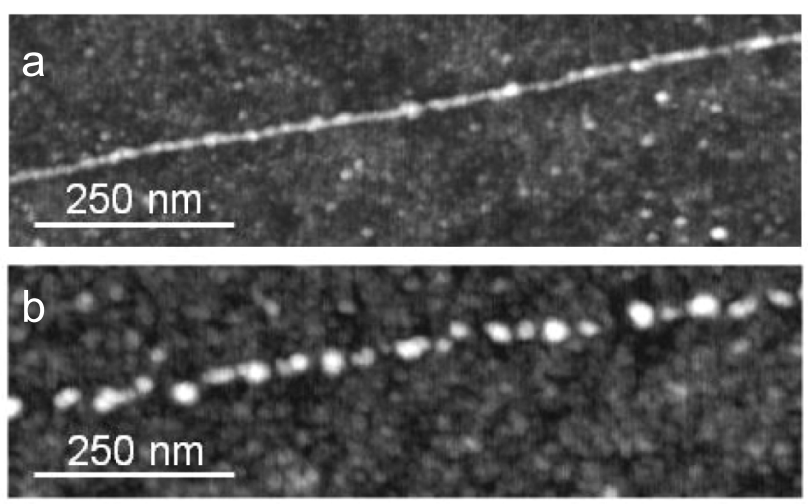

Fig. 5 AFM images of highly ordered assemblies of AN-AuNPs onto DNA molecules using method I (a) and II (b). The height scale is $5 \mathrm{~nm}$.

Phenaz-TMA-attached AuNPs (Phenaz-TMA/AuNPs) have also been prepared by photochemical reduction of $\mathrm{AuCl}_{4}^{-}$with $365 \mathrm{~nm}$ UV light in the presence of Phenaz-TMA. Since Phenaz-TMA (Fig. 6), which is the monomer unit of PPhenz-TMA, has an oxidation potential of $+1.245 \mathrm{~V}$ ( $v s$. $\mathrm{SHE}){ }^{76}$ it cannot directly reduce $\mathrm{AuCl}_{4}^{-}$, which has a reduction potential of $+1.002 \mathrm{~V}$ (vs. SHE). ${ }^{77}$ However, Phenaz-TMA excited by photoreaction has a greater reduction power, thus leading to spontaneous electron transfer from excited Phenaz-TMA (oxidation) to $\mathrm{AuCl}_{4}^{-}$(reduction). The photochemical formation of Phenaz-TMA/AuNPs was monitored by taking UV-vis absorption spectra as a function of the irradiation time (Fig. 7(a)). As the $\mathrm{HAuCl}_{4}$ solution was added, the absorption spectra of Phenaz-TMA resulted in a long tail on the long wavelength side $(350-400 \mathrm{~nm})$ of the peak. $\mathrm{Au}(\mathrm{III})$ gives stable complexes with $\mathrm{C}, \mathrm{N}, \mathrm{P}, \mathrm{S}$, or even O-donor ligands. ${ }^{78}$ The resulting long tail on $350-400 \mathrm{~nm}$ gives a suggestion about the formation of the $\mathrm{Au}$ (III)-Phenaz-TMA complex. Thus, an effective electron transfer for reducing $\mathrm{AuCl}_{4}{ }^{-}$can be achieved with $365 \mathrm{~nm}$ UV light. Consequently, 
<smiles>Cc1ccc2c(c1)N(CCC[N+](C)(C)C)c1ccc(C)cc1[Si]2(C)C</smiles>

PPhenaz-TMA<smiles>C[Si](C)(C)CCCN1c2ccc(Br)cc2[Si](C)(C)c2cc(Br)ccc21</smiles>

Phenaz-TMA

Fig. 6 Chemical structures of PPhenaz-TMA and Phenaz-TMA.
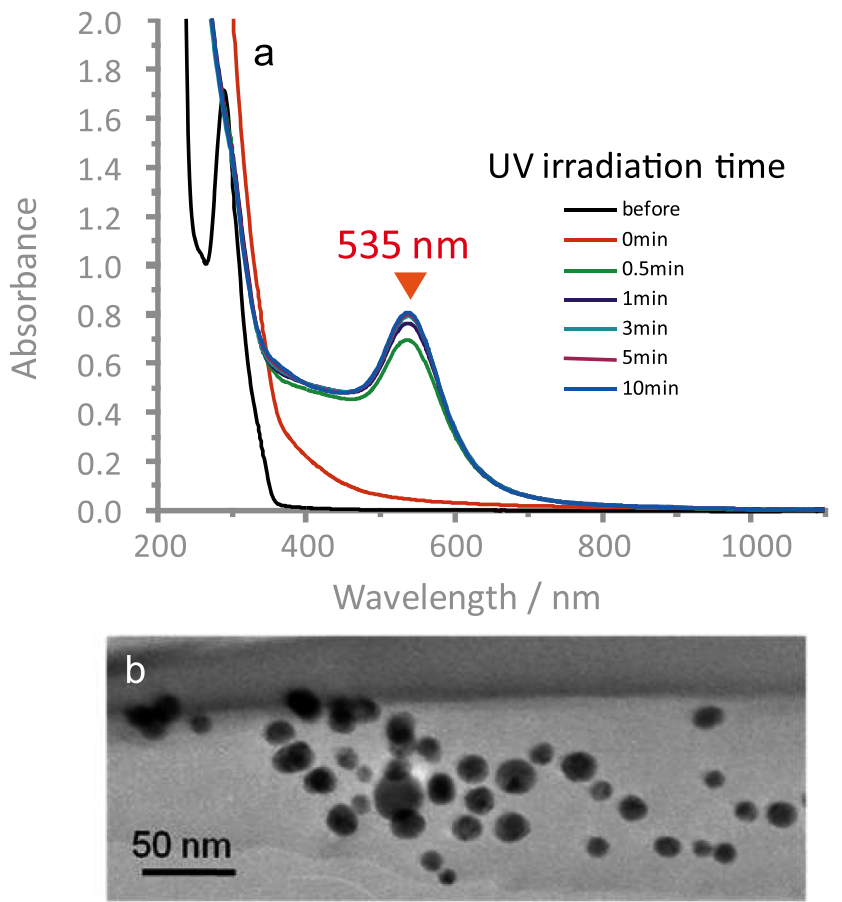

Fig. 7 Absorption spectra corresponding to the growth of the Phenaz-TMA/AuNPs by photochemical reduction (a). A $3 \mathrm{~mL}$ aqueous solution containing Phenaz-TMA $\left(150 \mathrm{nmol} \mathrm{mL}^{-1}\right)$ and $\mathrm{AuCl}_{4^{-}}\left(150 \mathrm{nmol} \mathrm{mL}^{-1}\right)$ was irradiated by $365 \mathrm{~nm} \mathrm{UV}$ light with $6 \times 8 \mathrm{~W}$. TEM image of prepared Phenaz-TMA/AuNPs.

an absorption peak at $530 \mathrm{~nm}$ was clearly observed and increased with increasing irradiation time. This absorption peak is attributed to the surface plasmon resonance (SPR) absorbance band of AuNPs. ${ }^{4-11}$ After a certain irradiation time (10 min in this case), the UV absorption spectrum reached a state of stagnation because of the completion of the photoreduction process. The TEM micrograph indicated that the prepared Phenaz-TMA/AuNPs were $5-20 \mathrm{~nm}$ in diameter (Fig. 7(b)).

The interaction between Phenaz-TMA/AuNPs and DNA was subsequently examined. Figure 8(a) illustrates a typical DNA titration of Phenaz-TMA/AuNPs solution. As DNA was gradually added, the SPR band intensity gradually increased, and the peak maximum shifted toward lower energy. The binding of Phenaz-TMA/AuNPs to DNA brings them close together, modifying their local environment and changing the SPR absorbance. ${ }^{69}$ Phenaz-TMA has alkylammonium salts on the $\mathrm{N}$ atom; additionally, it is oxidized during the preparation of AuNPs. Nanoparticles were electrostatically bound to the
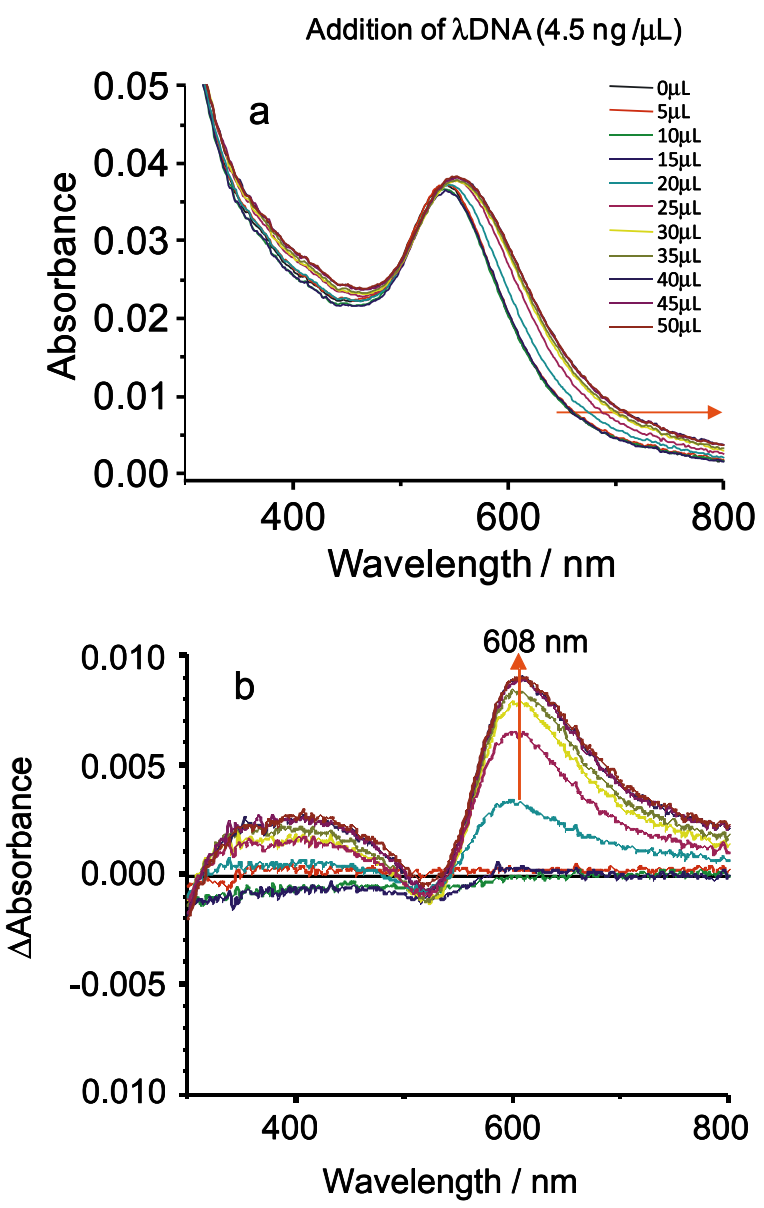

Fig. 8 Absorption spectra taken during titration of PhenazTMA/AuNPs with $\lambda$-DNA (a) and their difference spectra (b). DNA solution (4.5 $\left.\mathrm{ng} \mu \mathrm{L}^{-1}\right)$ was quantitatively added to Phenaz-TMA/AuNPs aqueous solution $\left(143 \mu \mathrm{g} \mathrm{mL}^{-1}\right)$.

negatively charged phosphate backbone of DNA by using Phenaz-TMA/AuNPs with a positively charged protecting monolayer. Figure 8(b) also shows differences in the spectra changes in Fig. 8(a). The absorption peak at $608 \mathrm{~nm}$ increases with an increase in DNA additions, which suggest the SPR of AuNPs assembled onto DNA molecules. Dark-field optical microscopy enables the observation of the LPR of single MNPs. In dark-field optical microscopy, only the light scattered by the structure under study is collected in the detection path, while the directly transmitted light is blocked using a dark-field condenser. Figure 9 presents scattered light images of Phenaz-TMA/AuNPs before and after attaching DNA. Dark-field light scattering images were acquired using a NIKON ECLIPSE 80i with a dark field condenser and a NIKON Power shot A640 digital camera. In general, individual sphere of AuNPs are observed as green particles originating from its LPR colors. ${ }^{79}$ Thus, the green particles of Phenaz-TMA/AuNPs in the figure are individually spherical. After DNA attachment, we observed a significant color change (reddish) in each of the particles along the DNA chains. As described above, when two or more particles are brought into a near-field interaction, the spectrum exhibits a clear red shift. ${ }^{180}$ This result also agrees with spectra changes at $608 \mathrm{~nm}$ shown in Fig. 8(b). Although each particle along the DNA cannot be resolved because of their diffraction-limited spots, the color change after DNA attachment strongly indicates that many particles were assembled along the DNA. 


\section{Fabrication and Patterning of Metallic Nanoarrays with Long Range Order}

Although DNA is an ideal 1D template to fabricate metallic nanoarrays, the length of prepared arrays essentially depends on that of the original DNA. For example, $\lambda$-DNA having a
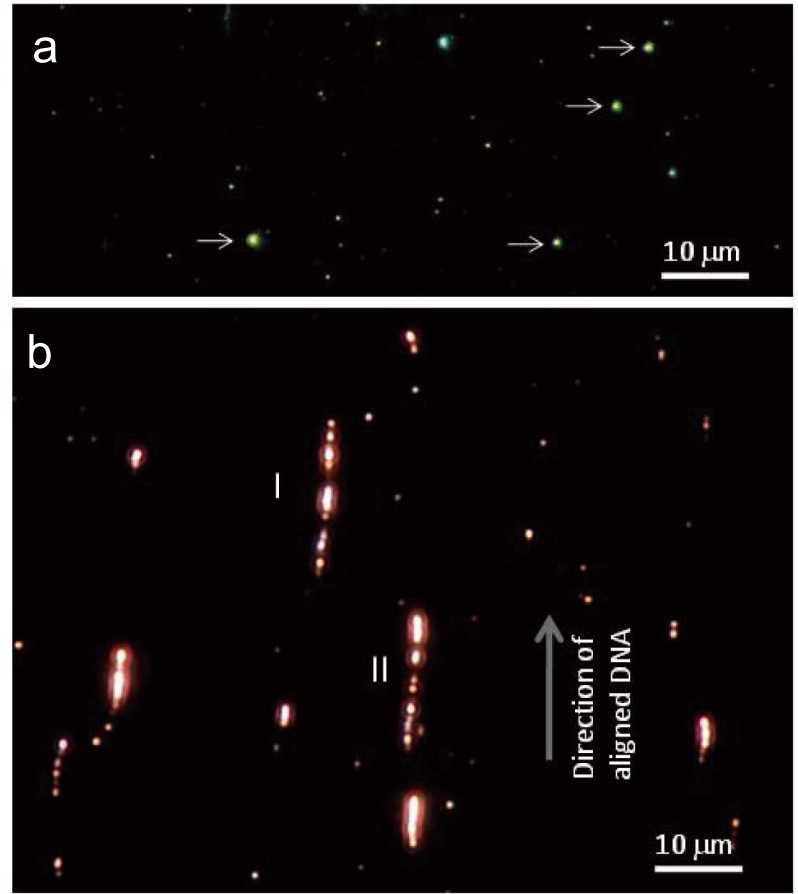

Fig. 9 Dark-field optical microscopy images of Phenaz-TMA/AuNPs before (a) and after DNA attaching (b). The arrow shows the individual sphere of Phenaz-TMA/AuNPs. Reddish lines (I) and (II) are Phenaz-TMA/AuNPs assembled on a single $\lambda$-DNA. theoretical length of $16.3 \mu \mathrm{m}$ is usually used as such a template. Since a 1D template with a greater geometrical aspect ratio leads to metallic nanoarrays with a larger scale, they can be easily manipulated during microscope observations, measuring their electrical and optical properties and sensing molecule interactions on them via SERS. Long DNA can be produced with some effort using a biochemical (enzymatic) technique, such as a polymerase chain reaction (PCR). In particular, rolling circle amplification (RCA $)^{81-83}$ can be used to produce a long single strand of DNA ( $>70 \mathrm{~kb}$ or more) ${ }^{84}$ which has a repeating sequence with a designed repeat unit on the order of 100 bases, and these repeat units can be addressed by hybridization to their complementary DNA sequence. The applicability of RCA for preparing DNA with periodic binding motifs has been demonstrated for arranging MNPs and binding proteins..$^{85,86}$ However, thus far, RCA and assembly reactions have been carried out solely in the liquid phase in a reaction tube. Such long DNA strands seem to be problematic for stretching and aligning on surfaces because of their intertwined state in solution.

\subsection{Preparation of longer metallic nanoarrays with DNA nanofibers}

Most recently, we have developed a simple method to create highly aligned DNA nanofibers on a surface. ${ }^{87}$ This method is based on the processes of solvent vapor-induced buildup and controlled drying front movement, and forms parallel aligned DNA nanofibers exceeding several hundred micrometers in length and $40 \mathrm{~nm}$ in diameter on a poly(dimethylsiloxane) (PDMS) sheet. Thus, this process leads to a DNA nanostrand that is much longer than the contour length of $\lambda$-DNA $(16.3 \mu \mathrm{m})$ and facilitates manipulating a single nanofiber under microscope observation, measuring its electrical and optical properties, and connecting it to electrode pads. Various MNPs could also be attached to such nanofibers, forming metallic nanoarrays with a longer scale.

The formation process for long metallic nanoarrays with DNA nanofibers is illustrated in Fig. 10.8 ${ }^{88}$ First, a mixture of Phenaz-TMA/AuNPs and $\lambda$-DNA in a TE buffer $(\mathrm{pH} 8)$ solution

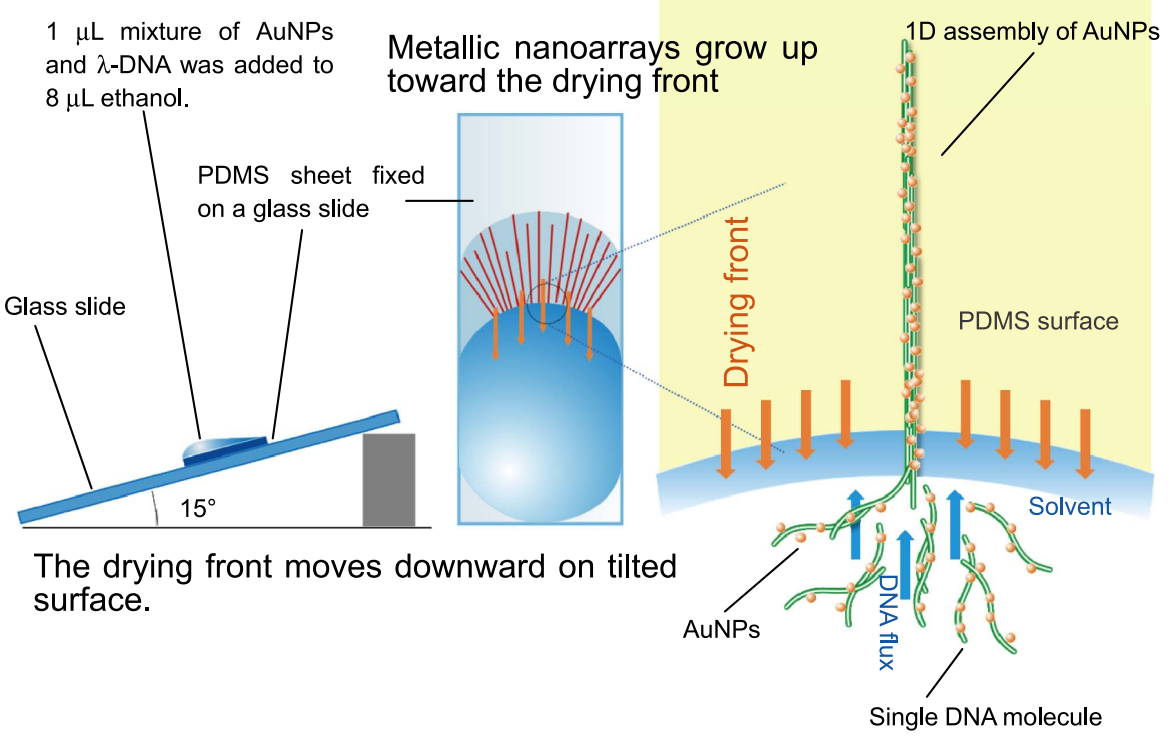

Fig. 10 Procedure of solvent vapor-induced assembles for generating metallic nanoarrays. Copyright 2009 The Japan Society for Analytical Chemistry. 

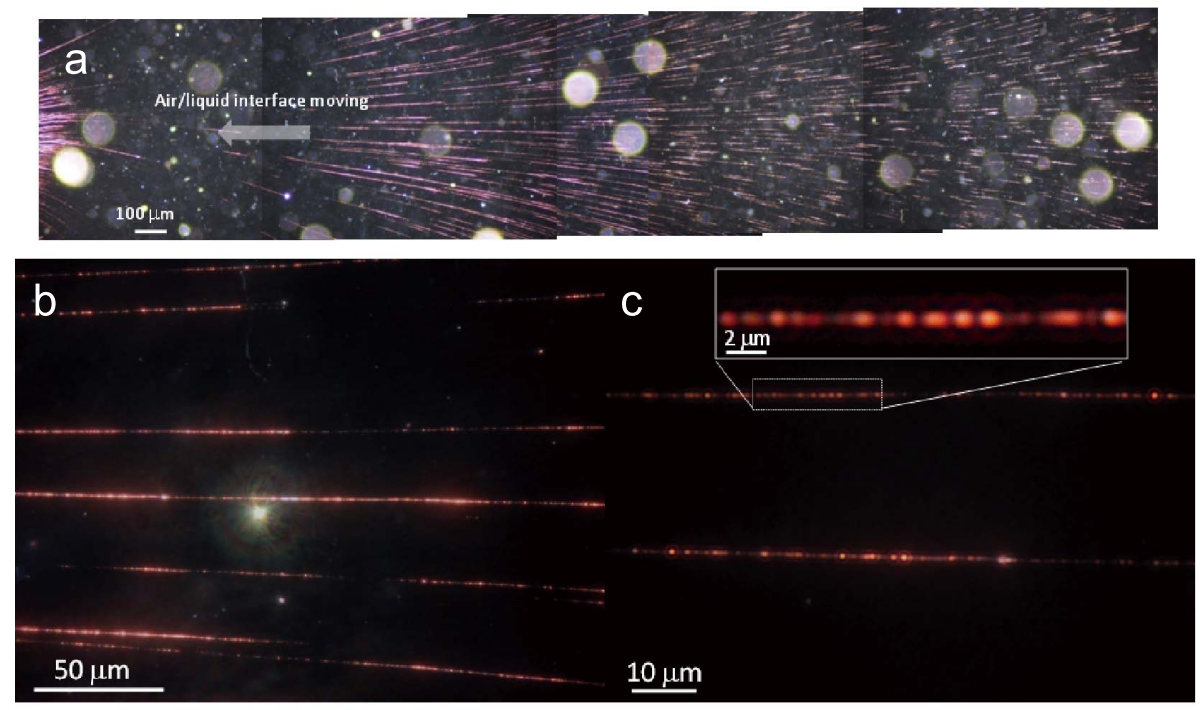

Fig. 11 Dark-field optical microscopy images of long metallic nanoarrays prepared with DNA nanofibers as tempalate: large area image of metallic nanoarrays (a). Enlarged image with $40 \times$ objective (b). Enlarged image with $100 \times$ objective (c). The drying front moved in a direction from right to left. Copyright 2009 The Japan Society for Analytical Chemistry.

was added to $8 \mu \mathrm{L}$ of ethanol. This solution was then deposited on a PDMS sheet. The PDMS sheet was tilted at $15^{\circ}$ during solvent evaporation to move the drying front downwards. Solvent evaporation leads to a decrease in the volume of the solution, leaving behind line patterns. Line patterns of metallic nanoarrays were formed when nanoparticles-attached DNA was continuously deposited at the highly concentrated finger positions. Figure 11 is a dark-field optical microscope image depicting metallic nanoarrays on a PDMS sheet prepared by the above method. Many reddish lines originating from the plasmon resonance of AuNPs assembled on DNA nanofibers are clearly observed. Although the curvature of the lines at the edges of the surface was due to the shape of the meniscus movement, they were aligned parallel to the moving drying front of the solvent. Metallic nanoarrays also exceed several hundred micrometers in length and can be made within $30 \mathrm{~min}$. Figure 12 presents an AFM image of metallic nanoarrays on the PDMS surface. The observed height (diameter) of metallic nanoarrays was 30 to $40 \mathrm{~nm}$ and was similar to that of bare DNA nanofibers. It is significant that AuNPs-attached DNA nanofibers have granulated nanostructures originating from AuNPs attachments along them. Enlarged optical and AFM images (Figs. 11(c) and 12(a)) indicate that AuNPs are nonuniformly attached on entire nanofibers. Further optimizations of the $\mathrm{pH}$ or ion strength in solution would achieve more efficient formations of metallic nanoarrays.

As described above, when MNPs are organized in closely spaced arrays, their LPR peak is shifted towards low energy, and an increase in the bandwidth is observed because the dielectric constant of the surrounding MNPs is increased. Such interparticle spacing therefore serves as highly localized light fields for enhancing SERS. Termed "hot spots" these highly confined fields also enable increasing fluorescent emission, albeit with more modest enhancement factors. Furthermore, since a 1D particle array can exhibit coupled modes due to near-field interactions between adjacent MNPs, it is possible to propagate electromagnetic waves (light) with a transverse confinement below the diffraction limit (plasmon waveguides).
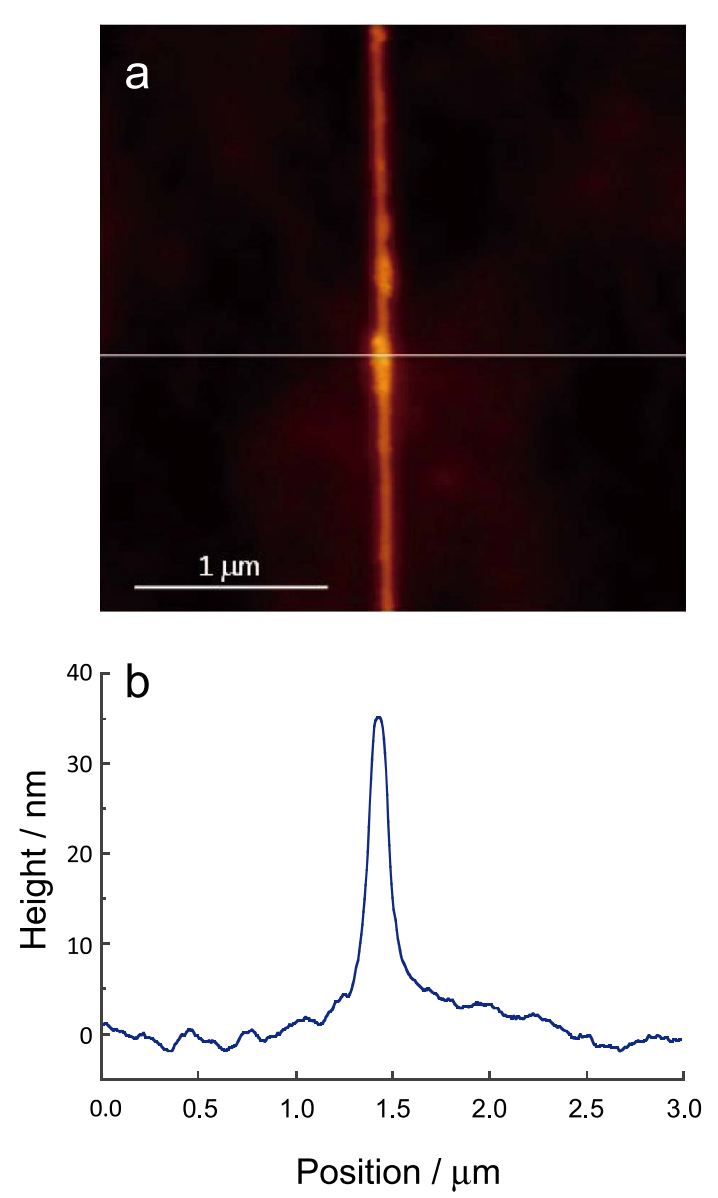

Fig. 12 (a) AFM images of metallic nanoarrays on the PDMS surface. (b) Cross-section profile along the white line on the AFM image (a). The drying front moved upward in the image. 

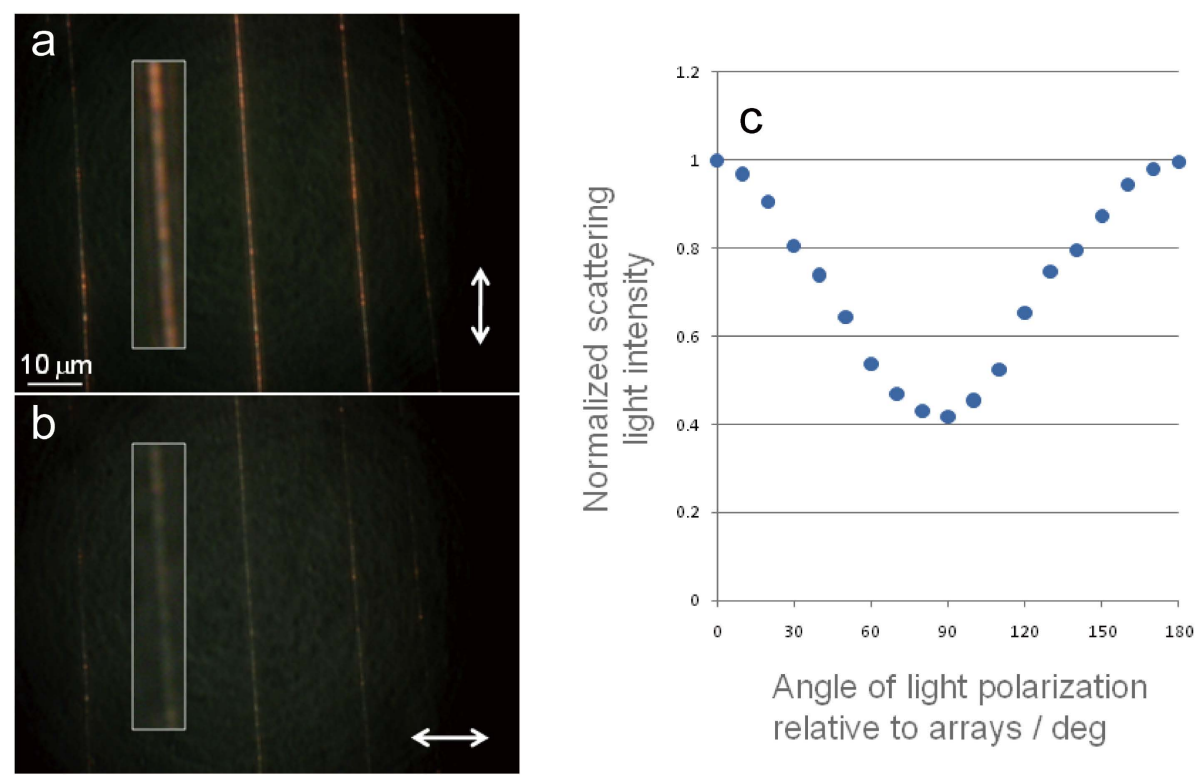

Fig. 13 Polarized dark-field optical microscopy images of metallic nanoarrays (a), (b). Arrows indicate the directions of polarized illumination. Scattering light intensities from metallic nanoarrays as a function of the angle between the arrays and the polarization (c). The polarization dependence of light scattering intensity in metallic nanoarrays. The angle is 0 degree when the illumination polarization is parallel to the arrays. Copyright 2009 The Japan Society for Analytical Chemistry.

For our several-hundred-micrometer-long 1D metallic nanoarray, such optical properties can be easily examined by microscopy. We observed dark-field optical images of our metallic nanoarrays under light polarization. The scattering light intensity of metallic nanoarrays is stronger when the light is polarized parallel to the arrays, while they are weaker for vertical polarization (Figs. 13(a) and (b)). Furthermore, the scattering intensity exhibited a cosine-like dependence on the polarization angle (Fig. 13(c)). The enhancement of the light field localized in gaps between MNPs also strongly depends on the incident light polarization. ${ }^{89}$ For a uniaxial alignment of ordered MNPs, polarization parallel to the uniaxial alignment enhances light field localization in gaps between MNPs, leading to strong light scattering from ordered MNPs. In contrast, vertical polarization to the uniaxial alignment does not enhance light field localization. Thus, the above result indicates that the alignment of AuNPs onto DNA nanofibers was preferentially uniaxial to the fiber axis. The intensity ratio was 2 , and this contrast could be further improved by increasing the uniformity of AuNPs-attaching to DNA nanofibers. Currently, to utilize our metallic nanoarrays as SERS chips for single-molecule detection and optical waveguides below the diffraction limit, further investigations, such as spectroscopy and light propagations on metallic nanoarrays, are being conducted.

\subsection{Preparation of $2 \mathrm{D}$ pattern of metallic nanoarray}

We have reported that stretched DNA molecules and nanofibers initially present on the PDMS sheet were transferred onto another surface using transfer-printing (TP). ${ }^{87,90}$ Furthermore, by repeating TP onto the same surface, it was also possible to realize a two-dimensional (2D) assembly of stretched DNA molecules and nanofibers. We also demonstrated the TP of 2D patterns of metallic nanoarrays onto surfaces. To assemble the patterns, we first transferred metallic nanoarrays onto a coverslip, and then overlapped the other PDMS sheet to which metallic nanoarrays were fixed onto the first one (Fig. 14(a)). a
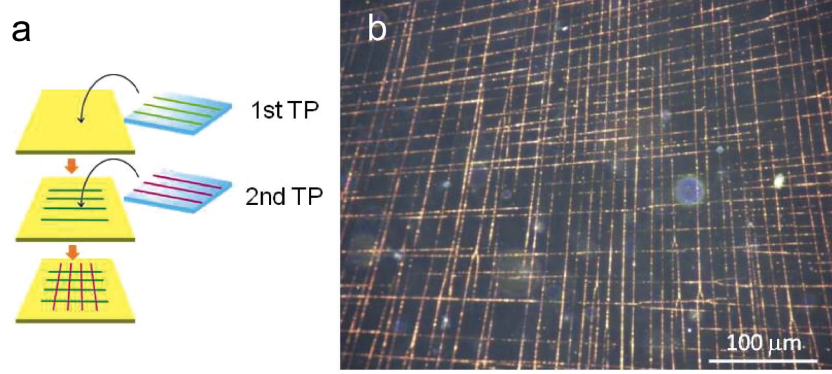

Fig. 14 2D pattern of metallic nanoarrays produced by repeating TP process. Procedure of TP process for creating 2D pattern of metallic nanoarrays (a). Dark-field optical micrograph of 2D metallic nanoarrays (b).

Since a glass surface has polar groups stronger than those on the hydrophobic surface of PDMS, such arrays containing hydrophilic groups could be easily transferred from a PDMS surface to a glass surface. The dark-field micrograph of 2D metallic nanoarray patterns is depicted in Fig. 14(b). The main advantages of TP are that metallic nanoarrays can be printed in the desired position on the substrate, and that they can be integrated on the same substrate by repeating the TP process, thus, allowing the integration and more complex patterning of metallic nanoarrays. By creating topological micropatterns on PDMS, it is also possible to achieve higher ordered patterns of metallic nanoarrays.

\section{Conclusion}

This review has summarized our recent works on methods for constructing metallic nanoarrays with a DNA template. Our 
methods first require DNA-stretching and fixation on surfaces. We have demonstrated that surface coating with polymers containing $\pi$-conjugation units enhance DNA-stretching and fixation on surfaces, due to the $\pi$ - $\pi$ interaction ( $\pi$-stacking) between aromatic amines in polymers and base pairs in DNA molecules. Such polymer-coating also enabled adequate optical microscopy and AFM observation of well-stretched DNA on conventional coverslip. The next step is DNA-attaching of MNPs. The AN-AuNPs prepared here strongly interacted with DNA. Two different assembly methods were carried out; consequently, continuous depositions and necklace-like depositions of AN-AuNPs along DNA molecules were achieved. The two approaches used in this study enabled different formations of metallic arrays of AN-AuNPs. Specifically, linear arrays of AN-AuNPs with interparticle spacing could be organized onto DNA molecules in a necklace-like formation. Furthermore, Phenaz-TMA/AuNPs was prepared by a photochemical reduction of $\mathrm{AuCl}_{4}{ }^{-}$with $365 \mathrm{~nm} \mathrm{UV}$ light in the presence of Phenaz-TMA. Phenaz-TMA/AuNPs also demonstrated strong interaction with DNA. UV-vis spectroscopy and dark-field optical microscopic observations revealed assemblies of Phenaz-TMA/AuNPs onto DNA and the LPR color changes involved. DNA nanofibers were useful for constructing metallic nanoarrays with a long range order. Furthermore, it was possible to create 2D patterns of metallic nanoarrays by the TP technique. Our methods summarized here do not require any special equipments, and should provide a useful system for investigating the electromagnetic (light) field localized between MNPs.

Many applications of metallic nanoarrays enhance light fields localized between MNPs. In the field of analytical chemistry, one promising application is optical sensors, and single molecule detection via SERS has been reported. Gold nanospheres, immobilized on top of 1-mercaptobenzoic acid monolayers, yielded a SERS enhancement factor of $10^{7}$, while other anisotropic shapes had enhancement factors of $10^{8}$ to $10^{9} .{ }^{91}$ Our highly anisotropic metallic nanoarrays therefore serve as highly localized light fields for enhancing SERS. In addition to SERS, surface enhanced fluorescence has also been reported for molecules near the surfaces of MNPs. ${ }^{92-95}$ While molecular fluorescence is quenched within $5 \mathrm{~nm}$ of the metal particle surface, at distances of $10 \mathrm{~nm}$ or greater, fluorescence is enhanced up to 100 -fold by the localized electric field and increased intrinsic decay of the fluorophore. ${ }^{92-95}$ Our metallic nanoarrays can also be restructured by the TP technique, and integrated into a desired position on various substrates, such as glass or silicon. Therefore, our method will advance the fabrication of optical sensor chips for single-molecule detection.

\section{Acknowledgements}

We would like to thank Dr. H. Hayashi (Nagoya Municipal Industrial Research Institute) for synthesizing PPhenaz, PPhenaz-TMA and Phenaz-TMA; Prof. H. Shiigi (Osaka Pref. Univ.) for preparing AN-AuNPs; and Prof. F. Iwata (Shizuoka Univ.) for SNOM observations. This work was supported by Iketani Science and Technology Foundation and Grants-in-Aid for Basic Sciences from the Ministry of Education, Science, Sports and Culture, Japan (Nos. 18710106 and 18310089).

\section{References}

1. S. A. Maier, "Plasmonics: Fundamentals and Application",
2007, Springer Science+Business Media LLC.

2. S. A. Maier, M. L. Brongersma, P, G. Kik, S. Meltzer, A. A. G. Requichia, and H. Atwater, Adv. Mater., 2001, 13, 1501.

3. S. A. Maier, P. G. Kik, H. A. Atwater, S. Meltzer, E. Harel, B. E. Koel, and A. A. Requichia, Nat. Mater., 2003, 2, 229.

4. R. Garrell, Anal. Chem., 1989, 61, 401A.

5. A. Campion and P. Kambhampati, Chem. Soc. Rev., 1998, 27, 241.

6. K. Kneipp, H. Kneipp, I. Itzkan, R. R. Dasari, and M. S. Feld, Chem. Rev., 1999, 99, 2957.

7. C. J. Murphy, T. K. Sau, A. M. Gole, C. J. Orendorff, J. Gao, L. Gou, S. E. Hunyadi, and T. Li, J. Phys. Chem. B, 2005, 109, 13857.

8. B. P. Khanal and E. R. Zubarev, Angew. Chem., Int. Ed., 2007, 46, 2195.

9. M. E. Stewart, C. R. Anderton, L. B. Thompson, J. Maria, S. K. Gray, J. A. Rogers, and R. G. Nuzzo, Chem. Rev., 2008, 108, 494.

10. P. K. Jain, X. Huang, I. H. El-Sayed, and M. A. El-Sayed, Acc. Chem. Res., 2008, 41, 1578.

11. S. Yamada, Anal. Sci., 2009, 25, 1059.

12. J. C. Love, L. A. Estroff, J. K. Kriebel, R. G. Nuzzo, and G. M. Whitesides, Chem. Rev., 2005, 105, 1103.

13. C. X. Yu and J. Irudayaraj, Anal. Chem., 2007, 79, 572.

14. J. Y. Chang, H. M. Wu, H. Chen, Y. C. Ling, and W. H. Tan, Chem. Commun., 2005, 1092.

15. Q. H. Wei, K. H. Su, S. Durant, and X. Zhang, Nano Lett., 2004, 4, 1067.

16. C. R. Barry, N. Z. Lwin, W. Zheng, and H. O. Jacobs, Appl. Phys. Lett., 2003, 83, 5527.

17. L. M. Demers, D. S. Ginger, S. J. Park, Z. Li, S. W. Chung, and C. A. Mirkin, Science, 2002, 296, 1836.

18. Y. Cui, M. T. Bjork, J. A. Liddle, C. Sonnichsen, B. Boussert, and A. P. Alivisatos, Nano Lett. 2004, 4, 1093.

19. Y. Yin, Y. Lu, and Y. Xia, J. Am. Chem. Soc., 2001, 123, 771.

20. S. W. Chung, G. Markovich, and J. R. Heath, J. Phys. Chem. B, 1998, 102, 6685.

21. R. P. Sear, S.-W. Chung, G. Markovich, W. M. Gelbart, and J. R. Heath, Phys. Rev. E, 1999, 59 , R6255.

22. P. Yang and F. Kim, Chem. Phys. Chem., 2002, 3, 503.

23. J. Huang, A. R. Tao, S. Connor, R. He, and P. Yang, Nano Lett., 2006, 6, 524.

24. W. A. Lopes and H. M. Jaeger, Nature, 2001, 414, 735.

25. F. Favier, E. C. Walter, M. P. Zach, T. Benter, and R. M. Penner, Science, 2001, 293, 2227.

26. M. P. Zach, K. H. Ng, and R. M. Penner, Science, 2000, 290, 2120.

27. I. Yamashita, Thin Solid Films, 2001, 393, 12.

28. R. A. Macmillan, C. D. Paavola, J. Howard, S. L. Chan, N. J. Zaluzec, and J. D. Trent, Nat. Mater., 2002, 1, 247.

29. S. W. Lee, C. Mao, C. E. Flynn, and A. M. Belcher, Science, 2002, 296, 892.

30. T. Scheibel, R. Parthasarathy, G. Sawicki, X.-M. Lin, H. Jaeger, and S. L. Lindquist, Proc. Natl. Acad. Sci. U. S. A., 2003, 100, 4527.

31. J. L. Coffer, S. R. Bigham, X. Li, R. F. Pinizzotto, Y. G. Rho, R. M. Pirtle, and I. L. Pirtle, Appl. Phys. Lett., 1996, 69, 3851 .

32. O. Harnack, W. E. Ford, A. Yasuda, and J. M. Wessels, Nano Lett., 2002, 2, 919.

33. K. Keren, M. Krueger, R. Gilad, G. Ben-Yoseph, U. Silvan, and E. Braun, Science, 2002, 72, 297.

34. M. Mertig, L. C. Ciacchi, R. Seidel, W. Pompe, and A. D. Vita, Nano Lett., 2002, 2, 841. 
35. M. G. Warnerd and J. E. Hutchison, Nat. Mater., 2003, 2 , 272.

36. T. Yonezawa, S. Onoue, and S. Kimizuka, Chem. Lett. 2002, 1172.

37. P. Alivisatos, K. P. Johnson, X. G. Peng, T. E. Wilson, C. J. Loweth, M. P. Bruchez, and P. G. Schultz, Nature, 1996, 382, 609.

38. R. Elghanian, R. C. Mucic, R. L. Letsinger, and C. A. Mirkin, Science, 1997, 277, 1078.

39. N. C. Seeman, Trends Biotechnol., 1999, 17, 437.

40. H. Yan, S. H. Park, G. Finkelstein, J. H. Park, and T. H. LaBean, Science, 2003, 301, 1882.

41. Y. He, T. Ye, M. Su, C. Zhang, A. E. Ribbe, W. Jinag, and C. Mao, Nature, 2008, 452, 198.

42. E. S. Andersen, M. Dong, M. M. Nielsen, K. Jahn, R. Subramani, W. Mamdouh, M. M. Golas, B. Sander, H. Stark, C. L. P. Oliveira, J. S. Pedersen, V. Birkedal, F Besenbacher, K. V. Gothelf, and J. Kjems, Nature, 2009 459, 73.

43. S. M. Douglas, H. Dietz, T. Liedl, B. Högberg, F. Graf, and W. M. Shih, Nature, 2009, 459, 414.

44. C. Erler and M. Mertig, J. Vac. Sci. Technol., B, 2009, 27, 939.

45. K. Otobe and T. Ohtani, Nucleic Acids Res., 2001, 29, e109.

46. H. Yokota, D. A. Nickerson, B. J. Trask, G. Van Den Engh, M. Hirst, I. Sadowski, and R. Aebersold, Anal. Biochem. 1998, 264, 158.

47. A. T. Woolley and R. T. Kelly, Nano Lett., 2001, 1, 345.

48. D. Bensimon, A. J. Simon, V. Croquette, and A. Bension, Phys. Rev. Lett., 1995, 74, 4754.

49. X. Michalet, R. Ekong, F. Fougerousse, S. Rousseaux, C. Schurra, N. Hornigold, M. Slegtenhorst, J. Wolfe, S. Povey, J. S. Beckmann, and A. Bensimon, Science, 1997, 277, 1518.

50. J. F. Allemand, D. Bensimon, L. Jullien, A. Bensiom, and V. Croquette, Biophys. J., 1997, 73, 2064.

51. A. Bensimon, A. Simon, A. Chiffaudel, V. Croquette, V. Heslot, and D. Bensimon, Science, 1994, 265, 2096.

52. Y. Okahata, T. Kobayashi, and K. Tanaka, Langmuir, 1996, 12, 1326.

53. P. A. Smith, C. D. Nordquist, T. N. Jackson, and T. S Mayer, Appl. Phys. Lett., 2000, 77, 1399.

54. X. Liang, K. J. Morton, R. H. Austin, and S. Y. Chou, Nano Lett., 2007, 7, 3774

55. M. Rief, H. Clausen-Schaumann, and H. E. Gaub, Nat. Struct. Biol., 1999, 6, 346.

56. J. Hu, Y. Zhang, H. Gao, M. Li, and U. Hartman, Nano Lett., 2002, 2, 55.

57. Y. Arai, R. Yasuda, K. Akashi, Y. Harada, H. Miyata, K. Kinosita Jr., and H. Itoh, Nature, 1999, 399, 446.

58. S. B. Smith, L. Finzi, and C. Bustamante, Science, 1992, 258,1122

59. H. Nakao, H. Hayashi, T. Yoshino, S. Sugiyama, K. Otobe, and T. Ohtani, Nano Lett., 2002, 2, 475.

60. T. Yamamoto, T. Shimizu, and E. Kurokawa, React. Funct. Polym., 2000, 43, 79.

61. D. Yang, T. Strode, H. P. Spielmann, A. H. J. Wang, and T. G. Burke, J. Am. Chem. Soc., 1998, 120, 2979.

62. Y.-F. Song and P. Yang, Polyhedron, 2001, 20, 501.

63. A. Kumar, M. Pattarkine, M. Bhadhade, A. B. Mandale, K. N. Ganesh, S. S. Datar, C. V. Dharmadhikari, and M. Sastry, Adv. Mater., 2001, 13, 341 .

64. J. Richter, R. Seidel, R. Kirsch, M. Mertig, W. Pompe, J. Plaschke, and H. K. Schackert, Adv. Mater., 2000, 12, 507.

65. W. E. Ford, O. Harnack, A. Yasuda, and J. M. Wessels, Adv
Mater., 2001, 13, 1793.

66. D. Zanchet, C. M. Micheel, W. J. Parak, D. Gerion, and A P. Alivisatos, Nano Lett., 2001, 1, 32

67. M. Mertig, L. C. Ciacchi, R. Seidel, W. Pompe, and A. De Vita, Nano Lett., 2002, 2, 841.

68. Y. Liu, W. Meyer-Zaika, S. Franzka, G. Schmid, M. Tsoli, and H. Kuhn, Angew. Chem., Int. Ed., 2003, 4, 95.

69. G. Wang and R. W. Murray, Nano Lett., 2004, 4, 95.

70. F. Patolsky, Y. Weizmann, O. Lioubashevski, and I. Willner, Angew. Chem., Int. Ed., 2002, 41, 2323.

71. G. Wang, J. Zhang, and R. W. Murray, Anal. Chem., 2002, 74,4320

72. H. Nakao, H. Shiigi, Y. Yamamoto, T. Tokonami, T. Nagaoka, S. Sugiyama, and T. Ohtani, Nano Lett., 2003, 3 , 1391.

73. H. Shiigi, Y. Yamamoto, N. Yoshi, H. Nakao, and T. Nagaoka, Chem. Commun., 2006, 4288.

74. H. Shiigi, R. Morita, Y. Yamamoto, S. Tokonami, H. Nakao, and T. Nagaoka, Chem. Commun., 2009, 3615.

75. H. Nakao, H. Hayashi, F. Iwata, H. Karasawa, S. Sugiyama, and T. Ohtani, Lamgmuir, 2005, 21, 7945.

76. H. Nakao, H. Hayashi, and K. Okita, Anal. Sci., 2001, 17, 545 .

77. A. J. Bard, R. Parsons, and J. Jordan, "Standard Potentials in Aqueous Solution", 1985, Dekker, New York.

78. A. Laguna, "Modern Supramolecular Gold Chemistry", 2008, WILEY-VHC Verlag GmbH \& Co. KGaA, Weinheim.

79. C. Sönnichsen, T. Franzl, T. Wilk, G. von Plessen, J. Feldmann, O. Wilson, and P. Mulvaney, Phys. Rev. Lett., 2002, 88, 77402 .

80. J. Prikulis, F. Svedberg, M. Käll, J. Enger, K. Ramser, M. Goksör, and D. Hanstorp, Nano Lett., 2004, 4, 115.

81. G. T. Walker, M. C. Little, J. G. Nadeau, and D. D. Shank, Proc. Natl. Acad. Sci. U. S. A., 1992, 89, 392.

82. A. Fire and S.-Q. Xu, Proc. Natl. Acad. Sci. U. S. A., 1995 92, 4641 .

83. H. Takahashi, K. Yamamoto, T. Ohtani, and S. Sugiyama, BioTechniques, 2009, 47, 609.

84. L. Blanco, A. Bernad, J. M. Lázaro, G. Martin, C Garmendia, and M. Salas, J. Biol. Chem., 1989, 264, 8935.

85. S. Beyer, P. Nickels, and F. C. Simmel, Nano Lett., 2005, 5, 719.

86. O. I. Wilner, S. Shimron, Y. Weizmann, Z.-G. Wang, and I. Willner, Nano Lett., 2009, 9, 2040.

87. H. Nakao, T. Taguchi, H. Shiigi, and K. Miki, Chem. Commun., 2009, 1858.

88. H. Nakao, H. Hayashi, H. Shiigi, and K. Miki, Anal. Sci., 2009, 25, 1175.

89. H. Xu, E. J. Bjerneld, M. Käll, and L. Börjesson, Phys. Rev. Lett., 1999, 83, 4357.

90. H. Nakao, M. Gad, S. Sugiyama, K. Otobe, and T. Ohtani, J. Am. Chem. Soc., 2003, 125, 7162

91. C. J. Orendorff, A. Gole, T. K. Sau, and C. J. Murphy, Anal. Chem., 2005, 77, 3261 .

92. K. Sokolov, G. Chumanov, and T. M. Cotton, Anal. Chem. 1998, 70, 3898.

93. J. Malicka, I. Gryzczynski, and J. R. Lakowicz, Anal. Chem., 2003, 75, 4408 .

94. A. Parfenov, I. Gryzczynski, J. Malicka, C. D. Geddes, and J. R. Lakowicz, J. Phys. Chem. B, 2003, 107, 8829.

95. J. R. Lakowicz, C. D. Geddes, I. Gryzczynski, J. Malicka, Z. Gryzczynski, K. Aslan, J. Lukomski, E. Matveera, J. Zhang, R. Badugn, and J. J. Huang, Fluorescence, 2004, 14,425 . 